\title{
Vascular Access Training for Resuscitative Endovascular Balloon Occlusion of the Aorta (REBOA) Placement: A Feasibility Study in Emergency Physicians
}

\author{
Suzanne M Vrancken MD ${ }^{1,2}$, Rayner CLA Maayen MD', Boudewijn LS Borger \\ van der Burg MD', Daniël Eefting MD PhD ${ }^{3,4}$, Thijs TCF van Dongen MD PhD ${ }^{1,5}$, \\ Ingvar TB Berg MD6, Mark W Bowyer MD7and Rigo Hoencamp MD PhD 1, 2, 4, \\ 'Department of Surgery, Alrijne Hospital, Leiderdorp, the Netherlands \\ 2Trauma Research Unit, Department of Surgery, Erasmus MC, University Medical Center Rotterdam, Rotterdam, the Netherlands \\ ${ }^{3}$ Department of Surgery, Haaglanden Medical Centre, The Hague, the Netherlands \\ ${ }^{4}$ Department of Surgery, Leiden University Medical Centre, Leiden, the Netherlands \\ ${ }^{5}$ Defense Healthcare Organization, Ministry of Defense, Utrecht, the Netherlands \\ ${ }^{6}$ Emergency Department, Haaglanden Medical Centre, The Hague, the Netherlands \\ ${ }^{7}$ Department of Surgery, Uniformed Services University of Health Sciences, and the Walter Reed National Military Medical \\ Center, Bethesda, Maryland, USA
}

\begin{abstract}
Background: Training vascular access skills for Resuscitative Endovascular Balloon Occlusion of the Aorta (REBOA) to emergency physicians (EPs) could contribute to better outcomes in patients with non-compressible truncal hemorrhage. This study aimed to determine whether a concise training program could train EPs to recognize anatomical structures and correctly visualize and identify the puncture site for percutaneous REBOA catheter placement. Methods: Our training program included basic anatomy and training in access materials for REBOA. Participants underwent expert-guided practice on each other and were then tested on key skills: the identification of anatomical structures, anatomy knowledge, technical skills for vascular access imaging with a handheld ultrasound, and time to identify adequate puncture site of the common femoral artery (CFA). Consultant vascular surgeons functioned as expert controls.

Results: Eleven EPs participated. They had a median overall technical skills score of 32.5 (27.0-35.0) and median time to identify the CFA puncture site of $52.9 \mathrm{~s}$ (35.6-63.7), which improved to $34.0 \mathrm{~s}(21.2-44.7)$ at the post-test $(Z=-2.756, P=0.006)$. Consultant vascular surgeons were significantly faster $(P=0.000)$.

Conclusions: EPs are capable of visualizing the femoral artery and vein within $1 \mathrm{~min}$. This speed improved rapidly after repetition. Our concise training program proved useful regardless of prior endovascular experience. This program, as a component of an expanded Endovascular Resuscitation and Trauma Management curriculum, in combination with realistic task training models (simulator, perfused cadaver, or live tissue) has the potential to provide effective training of the skills required to competently perform REBOA.
\end{abstract}

Keywords: Vascular Access; Ultrasound; Training; Aortic Balloon Occlusion; Emergency Physicians; REBOA

Received: 23 February 2021; Accepted: 9 March 2021

\section{Corresponding author:}

SM Vrancken MD, Department of Surgery, Alrijne Hospital, Simon Smitweg 1, 2353GA Leiderdorp, The Netherlands.

Email: smvrancken@alrijne.nl

Disclosure: The opinions or assertions contained herein are the private views of the authors and are not to be construed as official or reflecting the views of the Dutch Department of Defense, Dutch government, U.S. Department of Defense, the Uniformed Services University of the Health Sciences, or any other agency of the U.S. Government. Several authors are employees of the Dutch or U.S. Government.

(C) 2021 CC BY 4.0 - in cooperation with Depts. of Cardiothoracic/ Vascular Surgery, General Surgery and Anesthesia, Örebro University Hospital and Örebro University, Sweden 


\section{INTRODUCTION}

Although airway and breathing management are the first critical steps in the management of critically injured patients, controlling catastrophic bleeding is a major life-saving skill in trauma and vascular surgery. Recent experiences on the battlefields of Iraq and Afghanistan have validated the efficacy of tourniquets and massive transfusion protocols for managing extremity hemorrhage [1-3]. However, areas not amenable to tourniquet application, such as the neck, trunk and junctional regions, continue to represent challenges for prompt effective bleeding control. Given a number of recent mass casualty incidents from shootings and terrorist attacks, new concepts of truncal and junctional bleeding control need to be evaluated and possibly implemented.

Resuscitative Endovascular Balloon Occlusion of the Aorta (REBOA) is a technique in which a catheter with an occlusion balloon is advanced into the aorta and then inflated, thereby obstructing flow into the distal circulation as a temporary measure to control hemorrhage (Figure 1) $[4,5]$. The balloon can be inflated in either aorta zone 1 , between the left subclavian artery and celiac trunk, or zone 3 , between the distal renal artery and aortic bifurcation, depending on the level of injury. REBOA is an important component of the evolving hybrid practice of the Endovascular Resuscitation and Trauma Management (EVTM) of bleeding in trauma patients [6]. Previously, an EVTM workshop was developed as a training to introduce the principles of REBOA as an adjunct to the management of patients with massive hemorrhage from truncal or junctional injuries [7]. REBOA catheters have been used successfully in the hospital setting, combat environment, and even in the earliest phases of prehospital care [3]. However, there is an ongoing debate as to which professionals should obtain vascular access and perform REBOA. A recent joint statement by the American College of Surgeons Committee on Trauma (ACS COT) and the American College of Emergency Physicians (ACEP) states that emergency physicians (EPs) may perform REBOA after sufficient training [8].

An International Collaborative Workgroup has been developed to evaluate the safety and efficacy of REBOA and EVTM as a potential standard for the emergency care of selective patients. An important research question is whether it is feasible to train medical personnel with limited or no prior endovascular or surgical experience, including EPs, to perform such endovascular procedures. There are formal training curricula designed for physicians to train the skills necessary to perform REBOA. These include the Basic Endovascular Skills for Trauma (BEST $\left.{ }^{\mathrm{TM}}\right)$, the Endovascular Skills for Trauma
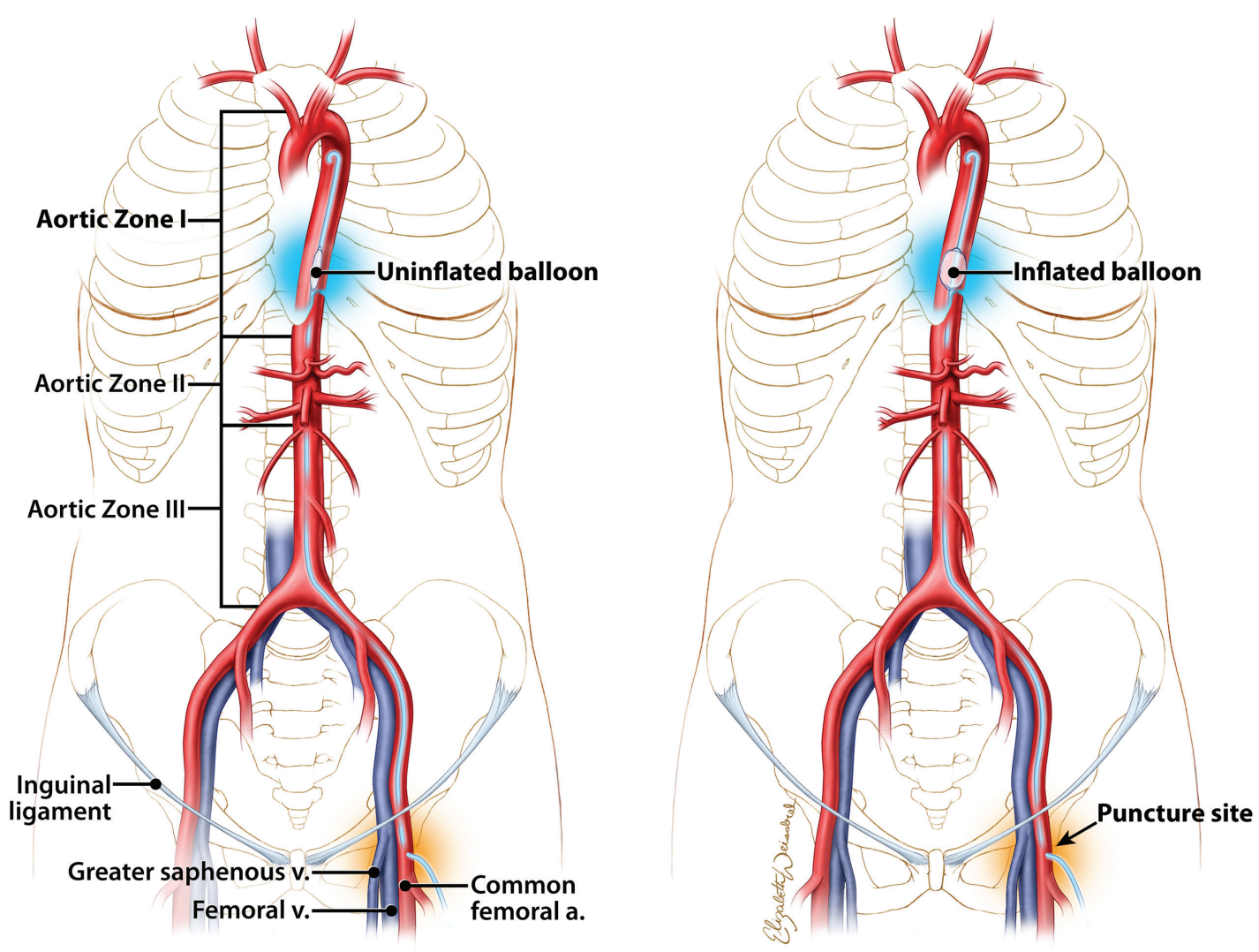

Figure 1 Schematic representation of REBOA balloon deployment in the aorta. 
and Resuscitation (ESTARS), and the EVTM courses. The EVTM International Collaboration Workgroup has recently reported on a comprehensive vascular access training program using a live-tissue simulator hybrid porcine model that can be used for femoral access and REBOA placement training in medical personnel with different levels of prior training [7].

In the Netherlands, EPs are integrated in emergency departments in all major trauma centers and many trauma units. They are often the first physicians providing emergency care. Vascular access training and REBOA placement for EPs could thereby contribute to better outcomes in the hemorrhagic patients they receive. Currently, endovascular procedures and the use of ultrasound to obtain vascular access are not part of the standard Dutch Emergency Physician training curriculum.

The primary aim of this study was to determine whether our concise training program could train EPs with limited or no experience to recognize anatomical structures and correctly visualize and identify the puncture site for the percutaneous placement of a REBOA catheter.

\section{METHODS}

\section{Participants}

All EPs from a Dutch level-I trauma center were approached to engage in our training program, and 11 signed up to participate. The participating EPs had varying prior experience with ultrasound imaging of the femoral artery and vein, and none of them had experience with REBOA. At their institution, the EPs receive patients from all medical disciplines and are generally the first physicians to provide emergency care. They are usually in charge of the management of trauma patients.

In this study, the participants, who were all healthy, also served as anatomy subjects, so they could practice the skills on each other. Three consultant vascular surgeons with extensive endovascular experience performed the same procedure, serving as expert controls.

\section{Curriculum}

Participants were asked to score themselves on their pre-existent general ultrasound skills and vascular access ultrasound skills on a scale from one (worst) to ten (best). A formalized concise training curriculum (30 min) comprised of basic anatomy of the femoral region, and knowledge of the access materials (the guide wire and introducer sheath) was presented. The details and instructions for use of the ER-REBOA ${ }^{\mathrm{TM}}$ Catheter (Prytime Medical; Boerne, Texas USA) were explained and demonstrated via an animated video (15 min) covering the steps necessary for deployment of the balloon in aortic zone 1 [4]. Participants were subsequently separated into buddy pairs and one trio for the

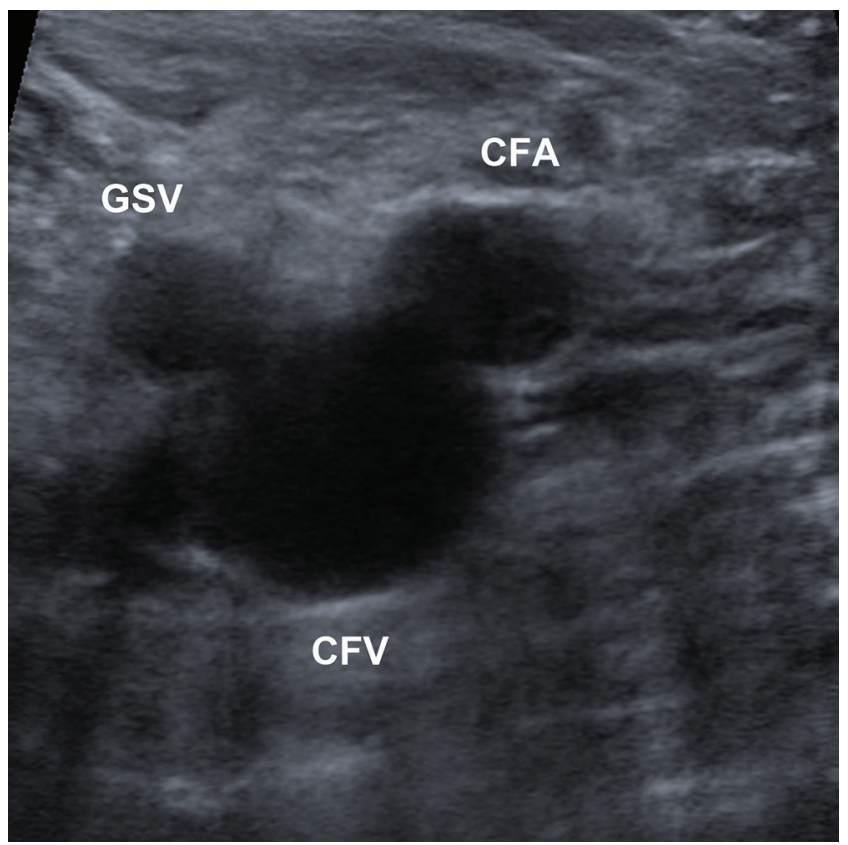

Figure 2 Transverse ultrasound image of the common femoral artery (CFA) and vein (CFV) and great saphenous vein (GSV), also known as Mickey Mouse sign.

training and test. They practiced (on each other) identification of the anatomical landmarks and ultrasound identification of the femoral vessels, guided by an experienced vascular surgeon or ultrasound specialist.

Key skills were as follows: (1) identification of anatomical structures, (2) anatomy knowledge, (3) technical skills for vascular access imaging using a handheld ultrasound and (4) time to identify adequate puncture site of the common femoral artery (CFA) with "Mickey Mouse sign" as seen in Figure 2.

A point-of-view GoPro® camera placed on a helmet was used for all participants, as well as one additional GoPro® camera positioned to achieve a full view of the subject and participant. After verbalizing every step of the procedure, video recording was commenced, and the actual test was started with registration of procedure time. As a post-test, all EPs performed the identical procedure a second time $2 \mathrm{~h}$ after additional endovascular training during this EVTM workshop.

\section{Scoring System}

Participants were evaluated using a modified checklist that was developed as part of a validation study for the Advanced Surgical Skills Exposures for Trauma (ASSET ${ }^{\mathrm{TM}}$ ) course $[9,10]$. This included technical and nontechnical skills, Global Rating Scale scores, errors, and time to achieve visualization of the "Mickey Mouse sign" (time from hand on ultrasound gel bottle to "Mickey Mouse sign"). Two evaluators (RH and TD) 
Table 1 (a) Self-rated pre-existent ultrasound skills of the emergency physicians and correlation with actual skills score. (b) Average results of the emergency physicians.

\begin{tabular}{|c|c|c|}
\hline a. Self-Rated Pre-Existent Ultrasound Skills & $\begin{array}{l}\text { Score }(n=11) \\
\text { Median }(I Q R)\end{array}$ & $\begin{array}{c}\text { Correlation } \\
\text { (Spearman's } \rho)\end{array}$ \\
\hline General ultrasound skills* & $6.0(3.0-7.0)$ & NA \\
\hline Vascular access ultrasound skills* & $5.0(3.0-6.0)$ & $\begin{array}{l}r_{s}=0.620 \\
P=0.042\end{array}$ \\
\hline b. Technical Skill & $\begin{array}{l}\text { Score }(n=11) \\
\text { Median }(I Q R)\end{array}$ & \\
\hline 01. Correctly identifies anatomical landmarks ${ }^{\dagger}$ & $4.0(3.0-4.0)$ & \\
\hline 02. Proceeds at appropriate pace with economy of movement ${ }^{\dagger}$ & $3.0(3.0-4.0)$ & \\
\hline 03. Communicates clearly and consistently ${ }^{\dagger}$ & $3.0(3.0-4.0)$ & \\
\hline 04. Follows a logical sequence for the procedure ${ }^{\dagger}$ & $4.0(3.0-4.0)$ & \\
\hline 05. Uses US probe in optimal orientation ${ }^{\dagger}$ & $4.0(3.0-4.0)$ & \\
\hline 06. Correctly identifies CFA & $1.0(1.0-1.0)$ & \\
\hline 07. Correctly identifies CFV ${ }^{\ddagger}$ & $1.0(1.0-1.0)$ & \\
\hline 08. Correctly identifies DFA & $1.0(1.0-1.0)$ & \\
\hline 09. Correctly identifies SFA ${ }^{\ddagger}$ & $1.0(1.0-1.0)$ & \\
\hline 10. Correctly identifies "Mickey Mouse" $\ddagger$ & $1.0(1.0-1.0)$ & \\
\hline 11. Identifies adequate puncture site $C F A^{\ddagger}$ & $1.0(1.0-1.0)$ & \\
\hline 12. Overall technical skills for introducing imaging CFA / CFV / DFA & $3.5(3.0-4.0)$ & \\
\hline 13. Overall understanding of the surgical anatomy of the femoral region ${ }^{\dagger}$ & $4.0(4.0-5.0)$ & \\
\hline Overall technical skills score & $32.5(27.0-35.0)$ & \\
\hline \multicolumn{3}{|l|}{$\begin{array}{l}\text { *Score ranging from } 1 \text { (worst)-10 (best). } \\
+ \text { Score ranging from } 0-5 \text {. } \\
\text { \# Score ranging from } 0-1 .\end{array}$} \\
\hline $\begin{array}{l}\text { CFA: common femoral artery; CFV: common femoral vein; IQR: interquartile ranges; DFA. } \\
\text { SFA: superficial femoral artery. }\end{array}$ & & \\
\hline
\end{tabular}

located in the same laboratory evaluated performance using a standardized script for data collection to ensure a reliable evaluation.

\section{Statistical Analysis}

Statistical analyses were performed in collaboration with an expert statistician, using the Statistical Package for the Social Sciences (SPSS®, Version 22, IBM Corporation, Armonk, New York). All baseline information from the subjects and subsequent follow-up data were registered in an electronic data file. The Wilcoxon signed rank test was used to analyze the procedure times. For all statistical analyses, a $P$ value equal or less than 0.05 was considered significant.

\section{Ethical Approval and Informed Consent}

The study was conducted under a protocol reviewed and approved by the Dutch Ministry of Defense and both the Institutional Review Board and Medical Ethics Committee of Alrijne Hospital, the Netherlands (NWMO 17-15, 17.409rt.tk). All participants completed an informed consent to participate in this effort.

\section{RESULTS}

Eleven EPs with varying ultrasound experience and no experience with REBOA participated in this study. They had a median self-evaluated pre-existent ultrasound skills score of $6.0(3.0-7.0)$ for general ultrasound skills and $5.0(3.0-6.0)$ for vascular access ultrasound skills (Table $1 a)$. The median overall technical skills score was 32.5 (27.0-35.0) (Table 1b). All EPs were able to identify the correct CFA puncture site, although two were unable to identify all appointed vascular structures correctly. The median global rating score for introducing imaging of the CFA, common femoral vein (CFV) and deep femoral artery (DFA) was 3.5 (3.0-4.0), and was $4.0(4.0-5.0)$ for overall understanding of the surgical anatomy of the femoral region (Table $1 b$ ). The median time from the start of the procedure to adequately identifying the puncture site of the CFA with "Mickey Mouse sign" was $52.9 \mathrm{~s}(35.6-63.7)$ on the first attempt, which significantly improved to $34.0 \mathrm{~s}(21.2-44.7)$ by the posttest $(Z=-2.756, P=0.006)$ (Table 2). Of $11 \mathrm{EPs}, 10$ were able to improve their procedure times on the second attempt. There was a significant correlation between self-evaluated pre-existent vascular access ultrasound skills and actual technical skills $\left(r_{\mathrm{s}}=0.620, P=0.042\right)$ 
Table 2 Time needed to identify adequate puncture site common femoral artery (CFA) with Mickey Mouse sign; emergency physicians $(n=11)$.

\begin{tabular}{lllll}
\hline Procedure Time & First Attempt (s) & Second Attempt (s) & Difference (s) & $\begin{array}{l}\text { Significance } \\
\text { (Wilcoxon Signed Rank) }\end{array}$ \\
\hline $\begin{array}{l}\text { Time total } \\
\text { Median (IQR) }\end{array}$ & $52.9(35.6-63.7)$ & $34.0(21.2-44.7)$ & -18.87 & $Z=-2.756, P=0.006$ \\
\hline IQR: interquartile ranges. & & & \\
\hline
\end{tabular}

Table 3 Procedure time emergency physicians (post-test) versus consultant vascular surgeons.

\begin{tabular}{lllll}
\hline Expert Level & Emergency Physicians $(n=11)(s)$ & Vascular Surgeons $(n=3)(s)$ & Difference $(s)$ & $\begin{array}{l}\text { Significance } \\
(\text { MWU-Test })\end{array}$ \\
\hline Time total & $33.9(12.6)$ & $9.8(5.2)$ & -24.13 & $\begin{array}{l}U=1.000, r=0.780, \\
P=0.000\end{array}$ \\
Mean (SD) & & & $P$ \\
\hline
\end{tabular}

MWU-test Mann-Whitney U-test; SD: standard deviation.

(Table 1a). The post-test procedure times of the consultant vascular surgeons (the expert controls) were significantly faster than those of the EPs $(P=0.000)$ (Table 3$)$.

\section{DISCUSSION}

This study provides evidence that it is feasible to train EPs with a concise training program in the use of ultrasound for femoral artery imaging to identify the correct puncture site for REBOA catheter placement, regardless of pre-existent endovascular experience and training. The EPs were able to identify the adequate puncture site of the CFA with satisfying procedure times after a curriculum of $30 \mathrm{~min}$. Procedure times improved significantly at the post-test, indicating that repetition of training is effective in improving procedure times. This finding is supported by the result that experienced consultant vascular surgeons were significantly faster in visualizing the CFA. Our training program could be the foundation of a comprehensive REBOA procedure training curriculum for health care providers who have limited or no endovascular experience.

Since the primary medical specialists, such as EPs, who assess patients with hemorrhagic trauma are usually not experienced in vascular procedures, it is important to show that it is feasible to train these primary care providers in possible life-saving skills, such as REBOA. Achieving vascular access during an early stage in the treatment of trauma patients is important, not only to enable possible REBOA or interventional radiology procedures, but also for continuous blood pressure monitoring, blood sampling, and (fluid) resuscitation. Trauma patients who arrive hemodynamically stable might deteriorate quickly, and obtaining vascular access can then be challenging. Adding a second "A" for vascular access to the well-known "ABCDE" mnemonic ("AABCDE") has therefore been proposed for modern trauma care [11]. In obtaining arterial groin access, identifying the correct CFA puncture site is an important skill, considering that cannulation of the smaller superficial femoral artery is a common mistake and may be associated with a greater risk of leg ischemia [11].

To our knowledge, this is the first study assessing the feasibility of training EPs with limited endovascular experience in identifying femoral structures for REBOA placement in living humans. Previous studies have focused on the training of medical and non-medical personnel with no prior ultrasound or endovascular experience in obtaining ultrasound-guided vascular access and placement of a REBOA catheter in a REBOA Access Task Trainer (RATT). In those studies, participants were able to perform REBOA with ultrasound-guided vascular access with acceptable procedure times where repetition improved procedure times significantly $[12,13]$. Another study focused on the feasibility of training surgical residents, EPs and surgeons with no or limited endovascular experience in placement of a REBOA catheter in a live-tissue simulator hybrid porcine model [7]. This study showed that residents and medical consultants with no prior endovascular experience were able to perform REBOA placement within acceptable procedure times and, again, that higher levels of training are associated with faster procedure times. In a different study, REBOA was placed without ultrasound-guided vascular access in 24 patients in tertiary medical centers in Japan [14]. In 22 of 24 patients, introducer sheaths and REBOA catheters were placed by EPs. In a case report, successful REBOA catheter placement was performed by an EP with the use of ultrasound [15].

Although previous studies have demonstrated that EPs are capable of localizing the femoral vein with ultrasound to diagnose deep vein thrombosis (DVT) or 
place a central venous line in the femoral vein $[16,17]$, the ultrasound techniques for these procedures and for REBOA vascular access imaging are not fully comparable. While DVT examinations can be performed in a stress-free environment without time pressure, REBOA vascular access imaging has to be performed quickly and under stressful circumstances. Furthermore, an average time of almost $15 \mathrm{~min}$ has been reported for ultrasound-guided central venous catheter placement in the femoral vein by EPs [16], while a mean time of less than $7 \mathrm{~min}$ has been reported for REBOA procedures conducted by trauma or vascular surgeons [18]. It is therefore important to show that EPs can identify the correct CFA puncture site in a limited amount of time. It has been demonstrated that over $50 \%$ of REBOA procedural times are attributed to obtaining CFA access [19].

A Japanese study reports that the use of REBOA is easy and safe for trained EPs [20]. This is an important finding considering that, to date, only a minority of REBOA procedures have been conducted by EPs [21,22]. Also, the current Dutch national training program of EPs does not cover endovascular procedures and the use of ultrasound to obtain vascular access, although some clinics choose to offer an internship that includes ultrasound-guided vascular access. It is desirable to proceed to an inguinal cut down when a physician fails to achieve percutaneous arterial access. This is challenging for providers with no sufficient training in vascular surgery and should be the focus of further training.

Obviously, there are limitations in this feasibility study. Some of the participants did have prior vascular ultrasound experience. Nevertheless, they also improved their procedure time after repetition. Furthermore, healthy EPs were used as a training model. This does not fully represent the reality of a bleeding hypotensive patient with possible obesity or peripheral vascular disease. However, it does provide standardization. Ideally, visualization and cannulation of the femoral artery using ultrasound would be trained on a hypotensive human (cadaver) model. It would be interesting to compare the EPs procedure times with procedure times of other consultants, such as vascular and trauma surgeons. Also, the training and testing environments were ideal and not stressful or austere, as would be expected in real-life situations. These variables can be added in a subsequent training phase and will be added in a prospective study with real trauma patients that is being planned. Further training is required using ultrasound in combination with a realistic moulage model and cadaver flow model. Such a setup is needed for percutaneous and open access training to achieve successful placement of REBOA in a hypotensive model with collapsed vessels.

\section{CONCLUSION}

This study shows that inexperienced EPs are, after training, capable of visualizing the femoral artery and vein within $1 \mathrm{~min}$. The speed of correct visualization improved rapidly after repetition. Our concise theoretical and practical training program proved useful regardless of prior endovascular experience and training. This training program, as a component of an expanded EVTM curriculum and in combination with realistic task training models (simulator, perfused cadaver, or live tissue) has the potential to provide effective training of the skills required to competently perform REBOA.

\section{Acknowledgements}

We acknowledge all participating emergency physicians for participation in the practical phase of the training. We would like to thank Ms. Elizabeth Weissbrod, MA, CMI, for the high-quality image contained in this manuscript.

\section{Ethics Statement}

(1) All the authors mentioned in the manuscript have agreed to authorship, read and approved the manuscript, and given consent for submission and subsequent publication of the manuscript.

(2) The authors declare that they have read and abided by the JEVTM statement of ethical standards including rules of informed consent and ethical committee approval as stated in the article.

\section{Conflicts of Interest}

The authors (SV, RM, BBB, DE, TD, IB, MB, RH) declare that there are no conflicts of interest that could inappropriately influence (bias) their work. We confirm that this submission has not been published elsewhere and is not under consideration by another journal. All authors have made substantial contributions to all of the following: (1) the conception and design of the study, or acquisition of data, or analysis and interpretation of data, (2) drafting the article or revising it critically for important intellectual content and (3) final approval of the version to be submitted.

\section{Funding}

This study was partly funded by the Alrijne Academy, the SZVK, the Dutch Ministry of Defense and the Karel Doorman Fund. No other support was provided.

\section{Author contributions}

SV, RM, BBB, DE, MB and RH prepared the study set-up. SV, RM, DE, TD, IB and RH included participants and performed the study during the EVTM training in The Hague and collected the data. SV and TD performed the statistical analyses. SV, RM, BBB, DE, IB, $\mathrm{MB}$ and $\mathrm{RH}$ prepared the manuscript. SV, RM, BBB, TD and $\mathrm{MB}$ prepared the tables and figures. SV, RM, BBB, 
$\mathrm{DE}, \mathrm{TD}, \mathrm{IB}, \mathrm{MB}$ and $\mathrm{RH}$ contributed to the final version of the paper.

\section{REFERENCES}

[1] Hoencamp R, Idenburg FJ, Hamming JF, Tan EC. Incidence and epidemiology of casualties treated at the Dutch role 2 enhanced medical treatment facility at multi national base Tarin Kowt, Afghanistan in the period 2006-2010. World J Surg. 2014;38(7):1713-8.

[2] van Dongen TT, Idenburg FJ, Tan EC, et al. Combat related vascular injuries: Dutch experiences from a role 2 MTF in Afghanistan. Injury. 2016;47(1):94-8.

[3] van Oostendorp SE, Tan EC, Geeraedts LM, Jr. Prehospital control of life-threatening truncal and junctional haemorrhage is the ultimate challenge in optimizing trauma care; a review of treatment options and their applicability in the civilian trauma setting. Scand J Trauma Resusc Emerg Med. 2016;24(1):110.

[4] Prytime Medical Devices. The ER-REBOATM Catheter's Demonstration Video. 2016. (Accessed 19 September, 2017, at https://www.youtube.com/watch?v=IPRbyWNlx3U).

[5] Stannard A, Eliason JL, Rasmussen TE. Resuscitative endovascular balloon occlusion of the aorta (REBOA) as an adjunct for hemorrhagic shock. J Trauma. 2011;71(6):1869-72.

[6] Hörer TM, Pirouzram A, Khan M, et al. Endovascular resuscitation and trauma management (EVTM) - practical aspects and implementation. Shock. 2020. doi: 10.1097/SHK.0000000000001529. Epub ahead of print.

[7] Borger van der Burg BLS, Horer TM, Eefting D, et al. Vascular access training for REBOA placement: a feasibility study in a live tissue-simulator hybrid porcine model. J R Army Med Corps. 2019;165(3):147-51.

[8] Brenner M, Bulger EM, Perina DG, et al. Joint statement from the American College of Surgeons Committee on Trauma (ACS COT) and the American College of Emergency Physicians (ACEP) regarding the clinical use of Resuscitative Endovascular Balloon Occlusion of the Aorta (REBOA). Trauma Surg Acute Care Open. 2018;3(1):e000154.

[9] Mackenzie CF, Garofalo E, Puche A, et al. Performance of vascular exposure and fasciotomy among surgical residents before and after training compared with experts. JAMA Surg. 2017;152(6):1-8.

[10] American College of Surgeons. ASSET: Advanced Surgical Skills for Exposure in Trauma. 2017. (Accessed 21 September, 2017, at https://www.facs.org/qualityprograms/trauma/education/asset).

[11] EVTM group. Top Stent - The art of EndoVascular hybrid Trauma and bleeding Management: Örebro University Hospital, c/o KärlThorax kliniken; 2017.
[12] Borger van der Burg BLS, Maayen RCLA, van Dongen TTCF, et al. Feasibility study vascular access and REBOA placement: From zero to hero. J Spec Oper Med. 2018;18(4):70-4.

[13] Borger van der Burg BLS, Vrancken SM, van Dongen TTCF, DuBose JJ, Bowyer MW, Hoencamp R. Feasibility study of vascular access and reboa placement in quick response team fire fighters. J Spec Oper Med. 2020;20(1):70-5.

[14] Sato R, Kuriyama A, Takaesu R, et al. Resuscitative endovascular balloon occlusion of the aorta performed by emergency physicians for traumatic hemorrhagic shock: a case series from Japanese emergency rooms. Crit Care. 2018;22(1):103.

[15] Lyon RF, Northern DM. REBOA by a non-surgeon as an adjunct during MASCAL. Am J Emerg Med. 2018; 36(6):1121 e5-e6.

[16] Ozakin E, Can R, Acar N, Baloglu Kaya F, Cevik AA. An evaluation of complications in ultrasound-guided central venous catheter insertion in the emergency department. Turk J Emerg Med. 2014;14(2):53-8.

[17] Blaivas M, Lambert MJ, Harwood RA, Wood JP, Konicki J. Lower-extremity Doppler for deep venous thrombosis-can emergency physicians be accurate and fast? Acad Emerg Med. 2000;7(2): 120-6.

[18] DuBose JJ, Scalea TM, Brenner M, et al. The AAST prospective Aortic Occlusion for Resuscitation in Trauma and Acute Care Surgery (AORTA) registry: Data on contemporary utilization and outcomes of aortic occlusion and resuscitative balloon occlusion of the aorta (REBOA). J Trauma Acute Care Surg. 2016;81(3): 409-19.

[19] Romagnoli A, Teeter W, Pasley J, et al. Time to aortic occlusion: It's all about access. J Trauma Acute Care Surg. 2017;83(6):1161-4.

[20] Shoji T, Tarui T, Igarashi T, et al. Resuscitative endovascular balloon occlusion of the aorta using a low-profile device is easy and safe for emergency physicians in cases of life-threatening hemorrhage. J Emerg Med. 2018; 54(4):410-8.

[21] Brenner M, Inaba K, Aiolfi A, et al. Resuscitative endovascular balloon occlusion of the aorta and resuscitative thoracotomy in select patients with hemorrhagic shock: early results from the American Association for the Surgery of Trauma's Aortic Occlusion in Resuscitation for Trauma and Acute Care Surgery Registry. J Am Coll Surg. 2018;226(5):730-40.

[22] Manzano-Nunez R, McGreevy D, Orlas CP, et al. Outcomes and management approaches of resuscitative endovascular balloon occlusion of the aorta based on the income of countries. World J Emerg Surg. 2020; $15(1): 57$. 